\title{
QUALITY AND YIELD OF DIFFERENT BASIL (OCIMUM BASILICUM L.) CULTIVARS WITH VARIOUS PLANTING AND CUTTING TIMES UNDER HOT MEDITERRANEAN CLIMATE
}

\author{
BARUT, M. ${ }^{1 *}-$ TANSI, L. S. ${ }^{1}-$ AKYUZ, A. M. ${ }^{1}-$ KARAMAN, $S .{ }^{2}$ \\ ${ }^{I}$ Department of Field Crops, Faculty of Agriculture, Çukurova University, 01330 Adana, Turkey \\ ${ }^{2}$ Department of Biology, Faculty of Science and Letter, Kahramanmaraş Sütçü İmam \\ University, 46100 Kahramanmaras, Turkey \\ *Corresponding author \\ e-mail: mbarut@cu.edu.tr; phone:+90-555-892-7794
}

(Received $27^{\text {th }}$ Feb 2021; accepted $14^{\text {th }}$ May 2021)

\begin{abstract}
The effects of global warming and climate change are felt around the world, and 2020 was the second hottest year ever. In the current study, basil cultivars (cinnamon, purple, lemon) were examined at different planting (1 April, 15 April, and 30 April) and cutting times (4 July and 25 August) under hot field conditions in Turkey. A wide range of significant variations were obtained among the cultivars for traits like plant height $(34.96-47.98 \mathrm{~cm})$, flower length $(5.93-10.22 \mathrm{~cm})$, number of flowers per plant (5.94-28.63), fresh herbage yield (2565.06-10787.27 $\left.\mathrm{kg} \mathrm{ha}^{-1}\right)$, dry herbage yield (431.29-1756.57 kg ha-1), leaves essential oil rate $(1.04-2.32 \mathrm{ml} / 100 \mathrm{~g})$, and flower essential oil rate $(1.20-2.61 \mathrm{ml} / 100 \mathrm{~g})$. Sixty-five components were found, representing $82.29-98.50 \%$ of the total essential oil. The components differed according to the leaves and flowers. Main essential oil components varied based on the cultivars; methyl cinnamate (22.03-44.08\%) for cinnamon basil, linalool (23.68-46.47\%) for purple basil, and citral (26.26-44.43\%) for lemon basil. Consequently, although more than two cuttings can be made depending on the region where basil is grown, in our region where climate change is felt more clearly, it did not show rapid growth and allowed just two cuttings.
\end{abstract}

Keywords: basil chemotypes, essential oil composition, climate change, morphoagronomic performance, medicinal and aromatic plants

\section{Introduction}

Basil (Ocimum basilicum L.) is a plant of the Lamiaceae family and commonly cultivated on the European and Asian continents for its distinctive herbal spicy and sweet aroma. Owing to essential oils, many species of the family especially of the Nepetoideae subfamily to which Ocimum belongs, are highly aromatic (Paton et al., 1999). Basil essential oils include a wide array of chemical components depending on variations in chemotypes, flower and leaf, and particularly the origin of the plant. Its essential oil has a number of chemotypes (linalool, methyl cinnamate, citral, methyl chavicol (estragole), eugenol, etc.). In addition, the aromatic and morphological characteristics of the plant are significantly affected by environmental conditions and agronomic techniques (Sims et al., 2014).

Plants are challenged by hostile conditions of temperature, drought and salinity which disrupt the growing processes (Suprasanna et al., 2016). For most of the plants, these are the main yield constraints of plant productivity. Moreover, today, global warming and climate change bring new challenges for plant adaptation in stressful environments. Climate data records give evidence of climate change key indicators; such as numerous reservoirs reached their lowest water storage levels in 15 years in Turkey (NASA, 2021). Plants rely on environmental cues such as changes in temperature or humidity to initiate 
physiological states similar to bud and leaf production. However, plant reproduction cannot occur as usual if flowers bloom before their pollinators are active, or if the fruit ripens after seed dispersers, such as birds, migrate to remote areas. It is inevitable that the fate of agriculture will change because of these challenges. Besides, extremely fluctative weather conditions in wider regions have a negative impact on agriculture (Beillouin et al., 2020). With these negative impacts, the temporary instability of agricultural production can endanger local and global food security. Additionally, the species most at risk are predicted to be those living in areas that will undergo the most rapid climate change (Gairola et al., 2010; Sharma et al., 2020; Shen et al., 2021). Thus, especially medicinal plant populations found in nature are threatened by changing temperature and precipitation regimes, and disruption of commensal relationships (Al-Huqail et al., 2020; Applequist et al., 2020).

In general, the literature indicates that an increase in stress levels (high temperatures, drought stress and high UV-8 levels) leads to higher levels of secondary metabolites (Hiltunen, 1999; Chang et al., 2005; Al-Huqail et al., 2020). These stress effects can act directly on important enzymes or indirectly by changing the allocation of organic and inorganic substances within the essential oil producing plant parts. Plants accumulate many small organic molecules to protect their cellular machinery against environmental stresses (Yancey, 2005; Chen and Jiang, 2010; Liang et al., 2013). Furthermore, if medicinal plants unexpectedly become $20 \%$ or $30 \%$ more potent, it may unconsciously hurt people using directly the plant. For instance, eugenol is a compound found in basil essential oil and can be toxic if present in high amounts (Markowitz et al., 1992). So, comprehensive chemical analysis is needed to identify any changes in levels of vital plant compounds.

Sowing or planting times will be a limiting factor for many countries in the following years due to climate change and global warming. Thus, it is vital to determine the effects of changing environments, which will help us in future climatic problems. Harvesting and planting times, especially in plants having essential oils, is also a critical procedure (Rao et al., 2011; Toncer et al., 2017). Hence, characterization of the quality is the management that can improve the productivity of plants by optimizing these characteristics. Basil allows more than one cutting in a growing season under proper conditions. However, little attention has been paid to a direct comparison among cuttings, cultivars, planting times and organs in this plant growing in the hot climate. Thus, our aim is to provide a detailed characterization of different Ocimum cultivars, which will give a brief insight into the quality traits of this worthy medicinal plant.

\section{Material and methods}

\section{Plant material and field experiment}

Ocimum basilicum var. cinnamon (cinnamon basil), Ocimum basilicum var. purpurascens (purple basil) and Ocimum basilicum var. citriodorum (lemon basil) used as plant material in this study. The seeds were obtained from the company of Zengarden. The field experiment was performed at the experimental area of the Faculty of Agriculture

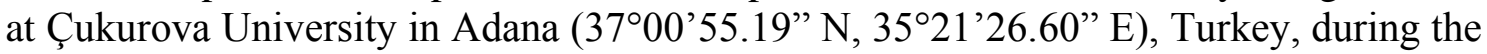
2020 growing seasons. The region normally has a typical Mediterranean climate with hot and dry summers, and moderate and rainy winters. However, in view of the long-term temperature and precipitation values, serious differences in air temperature and precipitation occurred in the year in which the experiment was carried out (Fig. 1). When 
we compared to 2019-2020 and long-term data, it can be easily observed large and hightemperature fluctuations and irregular precipitations. The soil at the site had a clay-loam texture and a low proportion $(1.11 \%)$ of organic matter. The soil was plowed to approximately $30-40 \mathrm{~cm}$ depth and then harrowed with a field cultivator to form. As plant fertilizers, $\mathrm{N}$ and $\mathrm{P}_{2} \mathrm{O}_{5}$ were distributed to the plots at a rate of $25 \mathrm{~kg} \mathrm{ha}^{-1}$ in the form of DAP (20-20-0), but potassium was not applied during the study as the soil was rich in K. The seeds were sown in plastic trays and transplanted into the field when they had 3-5 leaves with a $25 \mathrm{~cm}$ inter-row and intra-row spacing. The field experiment was arranged with three replicates using a split-plot design. The main plots were the planting times and sub-plots were the cultivars. The size of each plot was $9 \mathrm{~m}^{2}(3 \times 3 \mathrm{~m})$. Basil seedlings were planted the rows spacing at approximately $10 \mathrm{~cm}$ depth on 1 April, 15 April and 30 April. Ten plants were randomly selected from each plot to be used as both agronomic and quality parameters. Selected plants were marked and later these were used for measuring plants. The cutting times were 4 July for 1 st cutting and 25 August for 2nd cutting. In the study, agronomic traits such as plant height $(\mathrm{cm})$, number of branches per plant (pieces plant $\left.{ }^{-1}\right)$, flower length $(\mathrm{cm})$, fresh herbage yield $\left(\mathrm{kg} \mathrm{ha}^{-1}\right)$, fresh leaves yield $\left(\mathrm{kg} \mathrm{ha}^{-1}\right)$, fresh flowers yield $\left(\mathrm{kg} \mathrm{ha}^{-1}\right)$, dry herbage yield $\left(\mathrm{kg} \mathrm{ha}^{-1}\right)$, dry leaves yield $\left(\mathrm{kg} \mathrm{ha}^{-1}\right)$, dry flowers yield $\left(\mathrm{kg} \mathrm{ha}^{-1}\right)$, leaves essential oil rate $(\mathrm{ml} / 100 \mathrm{~g})$, flower essential oil rate $(\mathrm{ml} / 100 \mathrm{~g})$ were investigated.

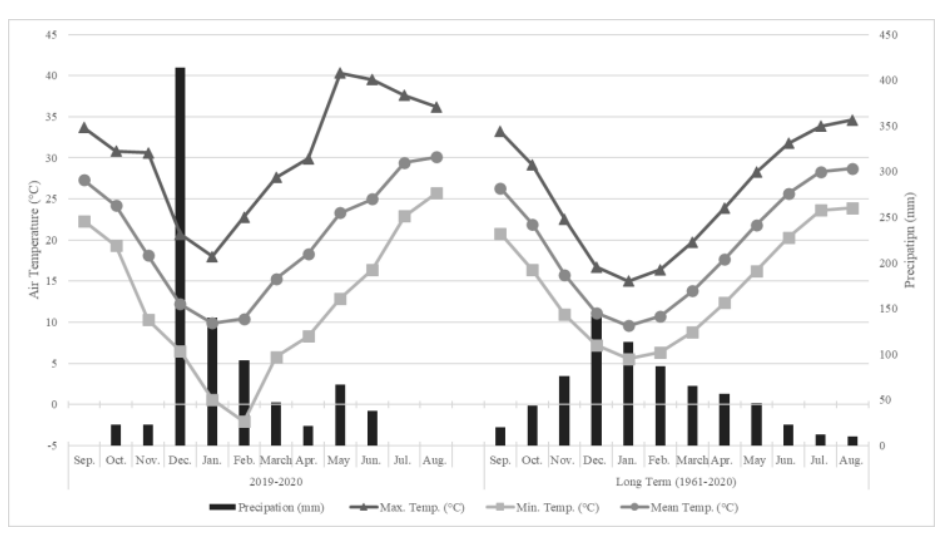

Figure 1. Meteorological data recorded during the field experiment and over the long term (1961-2020). The data was derived from the Meteorology Directorate of Adana, Turkey

\section{Essential oil extraction}

From each treatment, 25-30 g dried leaves and dried flower sample was weighed and placed in a glass balloon by putting $250 \mathrm{ml}$ distilled water and then placed into the Clevenger apparatus for hydrodistillation of essential oil for $2 \mathrm{~h}$. The essential oil extracted from the samples and accumulated on the water in the apparatus and the value was recorded (in $\mathrm{mL}$ ) from the graduated section of the apparatus. The oil rate was stated on a dry tissue weight basis. The chemical analyzes were done in triplicate.

\section{Gas chromatography / mass spectrometry (GC / MS) analysis}

GC-MS analyses were performed in the Department of Biology at Kahramanmaras Sutcu Imam University. $20 \mu \mathrm{l}$ essential oil was mixed with $0.5 \mathrm{ml}$ dichloromethane and $1 \mu \mathrm{l}$ mixture injected into the column. Essential oil components were investigated using an Agilent 5975C mass spectrometer in conjunction with the Agilent GC-6890 II series. 
The GC was equipped with an HP-88 capillary column (100 m x $250 \mu \mathrm{m} \times 0.20 \mu \mathrm{m}$ film thickness) and the carrier gas flow rate was $1.0 \mathrm{ml} / \mathrm{min}$. The oven temperature was held at $70{ }^{\circ} \mathrm{C}$ for $1 \mathrm{~min}$ and then increased from 70 to $220{ }^{\circ} \mathrm{C}$ at a rate of $10^{\circ} \mathrm{C} / \mathrm{min}$ and waited for $10 \mathrm{~min}$. Then increased to $230{ }^{\circ} \mathrm{C}$ at a rate of $10{ }^{\circ} \mathrm{C} / \mathrm{min}$ and held at $10 \mathrm{~min}$. The temperature of the injection part was $250{ }^{\circ} \mathrm{C}$. The mass spectrometer was operated in EI mode at $70 \mathrm{eV}$. The split ratio was 20:1. Mass range 35-400 m/z; scan speed (amu/s): 1000. The components were identified by mass spectra using Flavor2, HPCH1607 and Wiley7Nist05 libraries as reference compounds. Experimental workflow for essential oil component analysis was given in Fig. 2.

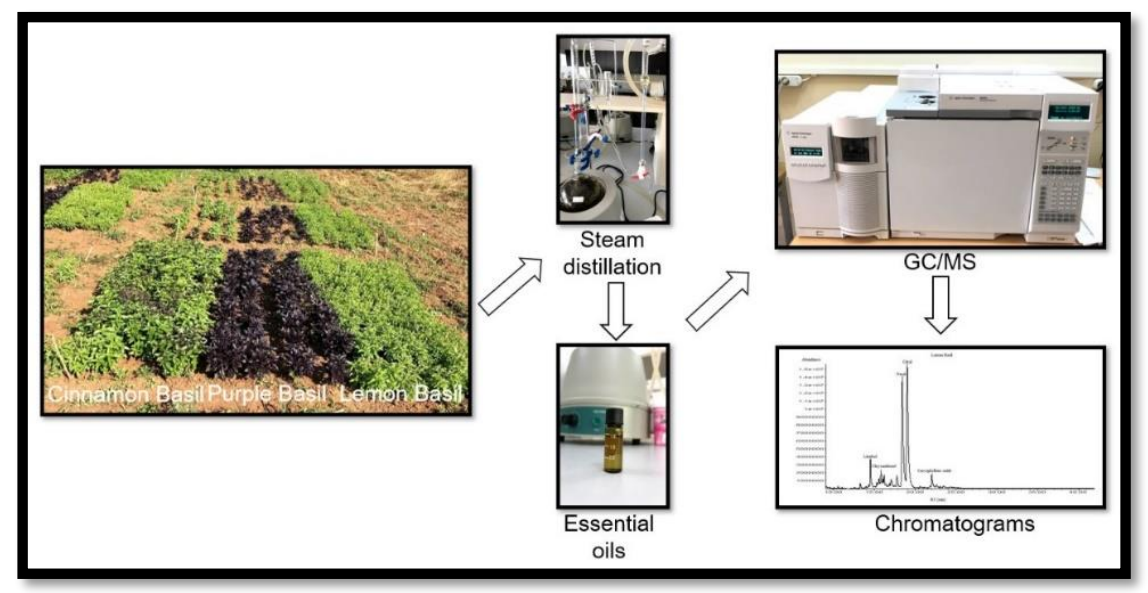

Figure 2. Experimental workflow from field to laboratory for essential oil component analysis

\section{Statistical analysis}

The data were analyzed according to a split-split plot design (planting dates $\times$ cultivars $\times$ cuttings) by an ANOVA model and principal components analysis on correlations using statistical software JMP ${ }^{\circledR}$ (version 14.0, SAS Institute Inc., Cary, NC, 1989-2019). Planting dates, cultivars, and cutting times were arranged as main plot, subplot, and sub-sub plot, respectively. It must be noted that the basic experimental design was based on the RCBD in a split-plot arrangement. Significantly different means were separated at $\mathrm{P}=0.05$ using the LSD (Least Significant Difference) test. The heat map was constructed using Flourish studio.

\section{Results and discussion}

\section{Agronomic traits}

Analysis of variance (ANOVA) was performed on 12 agronomic traits recorded in the field experiment to understand the effects of different planting times, different basil cultivars, and cuttings (Table 1). The studied plant agronomic traits revealed a wide range of variations in the evaluated basil cultivars. Cultivars were found highly significant for all the studied traits, while planting times revealed significant differences for half of the studied traits. Cuttings were found highly significant in all of the studied traits except for plant height and fresh leaves yield. Previous studies by Simon et al. (1992) and by Kothari et al. (2004) with basil, showed that the growth environment could have a great impact 
on the yield components. Correspondingly, our study, in which three cultivars of basil plants were subjected to different environments and the cutting times, showed an interaction of the growth environment and cultivars led to changes in studied traits. Overall means across the planting times, the cultivars and cuttings for agronomic traits are presented in Table 2.

Basil shows a varied range of genetic diversity in the agronomic traits like plant height, branches per plant, essential oil rate, and quality (Carović-Stanko et al., 2010; Rao et al., 2011; Patel et al., 2015). Plant height ranged from 41.00 to 45.34 with a mean of $42.95 \mathrm{~cm}$ among the planting dates. Minimum plant height and number of branches were recorded for purple basil, while the maximum was recorded in the lemon basil. Number of branches ranged from 8.90 to 10.03 with a mean of 9.48 pieces plant $^{-1}$ among the planting dates. Similar to what we detected in our study, the majority of other researches examining the effects of different applications on the plant height clearly showed parallel results; for cinnamon basil 43.30 to $46.50 \mathrm{~cm}$ (De Masi et al., 2006; Vieira and Simon, 2006), for purple basil 29.00 to $38.60 \mathrm{~cm}$ (Morales and Simon, 1996; Vieira and Simon, 2006; Nurzynska-Wierdak, 2007; Ekren et al., 2012), for lemon basil 27.30 to $48.8 \mathrm{~cm}$ (De Masi et al., 2006; Vieira and Simon, 2006; Nurzynska-Wierdak, 2007; Javanmardi and Ghorbani, 2012). As a matter of fact, Erşahin (2006) states that the plant height values of different basil populations are also different, varying between $37.13-82.07 \mathrm{~cm}$. These height differences were clearly observed in our study. While the cinnamon and lemon basil bloomed and formed large flowers, purple basil has just passed from the vegetative period to the generative period, and leaves were adversely affected by the increasing temperatures at this time.

Flower length ranged from 7.06 to 8.47 with a mean of $7.58 \mathrm{~cm}$ among the planting dates. Minimum flower length and number of flowers were recorded for purple basil, while the maximum was recorded in the lemon basil. Number of flowers ranged from 14.61 to 19.59 with a mean of 17.51 flowers plant $^{-1}$ among the planting dates. The values are in harmony with the findings obtained by Nurzynska-Wierdak (2007), Svecova and Neugebauerova (2010), Jaćimović et al. (2010), Nurzynska-Wierdak (2011), Tahhami Zarandi et al. (2014), Mirzajani et al. (2015), Moayedi et al. (2020). Nurzynska-Wierdak (2007) also found that lemon basil had more flowers than purple basil.

Fresh herbage yield ranged from $6,866.39$ to $8,309.35$ with a mean of 7,663.71 kg ha-1 among the planting dates. Minimum fresh herbage yield and fresh leaves yield was obtained with purple basil, as the maximum was exhibited for cinnamon basil. Fresh leaves yield ranged from $1,076.17$ to $1,418.96$ with a mean of $3,130.38 \mathrm{~kg} \mathrm{ha}^{-1}$ among the planting dates. Fresh flowers yield ranged from 1,719.48 to 3,000.44 with a mean of $2,164.84 \mathrm{~kg} \mathrm{ha}^{-1}$ among the planting dates. Minimum fresh flowers yield was recorded with purple basil, while the maximum was acquired for lemon basil. For flower yields, excluding the purple 1st cutting, the highest values were obtained in 30 April planting times in cinnamon and lemon basil, while there were no positive or negative trends in fresh leaf yields compared to planting times. When we compared the fresh herbage yield with the earlier studies, diverse results were found for the cultivars (Nurzynska-Wierdak, 2007; Majkowska-Gadomska et al., 2015; Cakmakci and Milton, 2019). These diverse results are thought to be due to differences between plant material and treatments as well as climatic changes. Walters and Currey (2019) indicated that the fresh yield in basil increased with the temperature to $29^{\circ} \mathrm{C}$, but then decreased to $35^{\circ} \mathrm{C}$ in the greenhouse. In our study, when the maximum temperature was above $35^{\circ} \mathrm{C}$ in the cutting times, it had a negative effect, especially for purple basil. 
Table 1. The analysis of variance results related to traits

\begin{tabular}{|c|c|c|c|c|c|c|c|c|c|c|c|c|c|}
\hline Factor & DF & $\begin{array}{l}\text { Plant height } \\
\text { (cm) }\end{array}$ & $\begin{array}{c}\text { Number of } \\
\text { branches } \\
\text { (pieces plant }^{-1} \text { ) }\end{array}$ & $\begin{array}{l}\text { Flower } \\
\text { length } \\
(\mathrm{cm})\end{array}$ & $\begin{array}{c}\text { Number of } \\
\text { flowers } \\
\text { (flowers } \\
\text { plant }^{-1} \text { ) }\end{array}$ & $\begin{array}{c}\text { Fresh } \\
\text { herbage } \\
\text { yield } \\
\left(\mathbf{k g ~ h a}^{-1}\right)\end{array}$ & $\begin{array}{c}\text { Fresh } \\
\text { leaves } \\
\text { yield } \\
\left(\mathbf{k g ~ h a}^{-1}\right)\end{array}$ & $\begin{array}{c}\text { Fresh } \\
\text { flowers } \\
\text { yield } \\
\left(\mathbf{k g ~ h a}^{-1}\right)\end{array}$ & $\begin{array}{c}\text { Dry } \\
\text { herbage } \\
\text { yield } \\
\left(\mathbf{k g ~ h a}^{-1}\right)\end{array}$ & $\begin{array}{c}\text { Dry } \\
\text { leaves } \\
\text { yield } \\
\left(\mathbf{k g ~ h a}^{-1}\right)\end{array}$ & $\begin{array}{c}\text { Dry } \\
\text { flowers } \\
\text { yield } \\
\left(\mathrm{kg} \mathrm{ha}^{-1}\right)\end{array}$ & \begin{tabular}{|c|} 
Leaves \\
essential \\
oil rate \\
$(\mathrm{ml} / \mathbf{1 0 0} \mathrm{g})$
\end{tabular} & \begin{tabular}{|c} 
Flower \\
essential \\
oil rate \\
(ml/100 \\
g)
\end{tabular} \\
\hline Planting times & 2 & ns & ns & ns & ns & $* *$ & $* *$ & $* *$ & $*$ & ns & $* *$ & ns & $* *$ \\
\hline Cultivars & 2 & $* *$ & $* *$ & $* *$ & $* *$ & $* *$ & $* *$ & $* *$ & $* *$ & $* *$ & $* *$ & $* *$ & $* *$ \\
\hline Cuttings & 1 & ns & $* *$ & $* *$ & $* *$ & $* *$ & ns & $* *$ & $* *$ & $* *$ & $* *$ & $* *$ & $*$ \\
\hline Planting times x Cultivars & 4 & ns & $*$ & ns & $* *$ & $* *$ & $*$ & $* *$ & ns & ns & $* *$ & ns & ns \\
\hline Planting times $\mathrm{x}$ Cuttings & 2 & ns & $* *$ & ns & $*$ & $* *$ & $* *$ & $\mathrm{~ns}$ & ns & ns & $* *$ & $* *$ & $* *$ \\
\hline Cultivars x Cuttings & 2 & * & $* *$ & ns & $* *$ & $\mathrm{~ns}$ & $* *$ & $* *$ & $\mathrm{~ns}$ & $\mathrm{~ns}$ & $* *$ & $* *$ & $* *$ \\
\hline $\begin{array}{c}\text { Planting times x Cultivars } \mathrm{x} \\
\text { Cuttings }\end{array}$ & 4 & ns & ns & ns & ns & $* *$ & $*$ & $* *$ & $* *$ & $\mathrm{~ns}$ & $* *$ & $*$ & $* *$ \\
\hline
\end{tabular}

DF: Degrees of Freedom. ns: not significant, *: $\mathrm{P}<0.05, * *$ : $\mathrm{P}<0.01$ 
Table 2. Yield performance of different basil cultivars

\begin{tabular}{|c|c|c|c|c|c|c|c|c|c|c|c|c|}
\hline Factor & $\begin{array}{c}\text { Plant height } \\
(\mathrm{cm})\end{array}$ & $\begin{array}{c}\text { Number of } \\
\text { branches } \\
\left(\text { pieces plant }^{-1}\right)\end{array}$ & $\begin{array}{l}\text { Flower } \\
\text { length } \\
(\mathrm{cm})\end{array}$ & $\begin{array}{c}\text { Number of } \\
\text { flowers } \\
\text { (flowers plant }{ }^{-1} \text { ) }\end{array}$ & $\begin{array}{c}\text { Fresh } \\
\text { herbage } \\
\text { yield } \\
\left(\mathbf{k g ~ h a}^{-1}\right) \\
\end{array}$ & $\begin{array}{c}\text { Fresh leaves } \\
\text { yield } \\
\left(\mathrm{kg} \mathrm{ha}^{-1}\right)\end{array}$ & $\begin{array}{c}\text { Fresh } \\
\text { flowers } \\
\text { yield } \\
\left(\mathbf{k g ~ h a}^{-1}\right) \\
\end{array}$ & $\begin{array}{c}\text { Dry } \\
\text { herbage } \\
\text { yield } \\
\left(\mathbf{k g ~ h a}^{-1}\right) \\
\end{array}$ & $\begin{array}{c}\text { Dry flowers } \\
\text { yield } \\
\left(\mathrm{kg} \mathrm{ha}^{-1}\right)\end{array}$ & $\begin{array}{c}\text { Dry leaves } \\
\text { yield } \\
\left(\mathbf{k g ~ h a}^{-1}\right)\end{array}$ & $\begin{array}{c}\text { Leaves } \\
\text { essential oil } \\
\text { rate } \\
(\mathrm{ml} / 100 \mathrm{~g}) \\
\end{array}$ & $\begin{array}{c}\text { Flower } \\
\text { essential oil } \\
\text { rate } \\
(\mathrm{ml} / \mathbf{1 0 0} \mathrm{g}) \\
\end{array}$ \\
\hline \multicolumn{13}{|l|}{ Planting times } \\
\hline 1 April & 42.51 & 8.90 & 7.23 & 18.32 & $8309.35 \mathrm{a}$ & $3562.60 \mathrm{a}$ & $1774.59 \mathrm{~b}$ & $1405.23 \mathrm{a}$ & $335.47 \mathrm{~b}$ & 565.20 & 1.78 & $2.04 \mathrm{a}$ \\
\hline 15 April & 41.00 & 9.50 & 7.06 & 14.61 & $6833.39 \mathrm{c}$ & $3138.54 \mathrm{~b}$ & $1719.48 \mathrm{~b}$ & $1076.17 \mathrm{~b}$ & $291.95 \mathrm{~b}$ & 464.86 & 1.89 & $1.89 \mathrm{a}$ \\
\hline 30 April & 45.34 & 10.03 & 8.47 & 19.59 & $7848.38 \mathrm{~b}$ & $2690.00 \mathrm{c}$ & $3000.44 \mathrm{a}$ & $1418.96 \mathrm{a}$ & $612.79 \mathrm{a}$ & 403.29 & 1.88 & $1.43 \mathrm{~b}$ \\
\hline $\operatorname{LSD}(\% 5)$ & ns & ns & ns & ns & 387.30 & 303.10 & 269.80 & 251.40 & 124.50 & ns & ns & 0.18 \\
\hline \multicolumn{13}{|l|}{ Cultivars } \\
\hline Cinnamon basil & $47.98 \mathrm{a}$ & $9.84 \mathrm{~b}$ & $6.60 \mathrm{~b}$ & $17.96 \mathrm{~b}$ & $10787.27 \mathrm{a}$ & $4631.94 \mathrm{a}$ & $2312.66 \mathrm{~b}$ & $1756.57 \mathrm{a}$ & $371.37 \mathrm{~b}$ & $734.52 \mathrm{a}$ & $2.18 \mathrm{a}$ & $2.61 \mathrm{a}$ \\
\hline Purple basil & $34.96 \mathrm{~b}$ & $5.76 \mathrm{c}$ & $5.93 \mathrm{~b}$ & $5.94 \mathrm{c}$ & $2565.06 \mathrm{~b}$ & $1166.12 \mathrm{c}$ & $479.64 \mathrm{c}$ & $431.29 \mathrm{~b}$ & $98.90 \mathrm{c}$ & $188.05 \mathrm{c}$ & $1.04 \mathrm{~b}$ & $1.55 \mathrm{~b}$ \\
\hline Lemon basil & $45.92 \mathrm{a}$ & $12.83 \mathrm{a}$ & $10.22 \mathrm{a}$ & $28.63 \mathrm{a}$ & $9638.79 \mathrm{a}$ & $3593.08 \mathrm{~b}$ & $3702.21 \mathrm{a}$ & $1712.50 \mathrm{a}$ & $769.94 \mathrm{a}$ & $510.78 \mathrm{~b}$ & $2.32 \mathrm{a}$ & $1.20 \mathrm{~b}$ \\
\hline $\operatorname{LSD}(\% 5)$ & 3.50 & 1.07 & 1.02 & 2.45 & 1540.00 & 636.80 & 535.30 & 245.40 & 131.10 & 107.00 & 0.25 & 0.35 \\
\hline \multicolumn{13}{|l|}{ Cuttings } \\
\hline 1st Cutting & 43.89 & $7.10 \mathrm{~b}$ & $6.90 \mathrm{~b}$ & $21.82 \mathrm{a}$ & $8645.10 \mathrm{a}$ & 3170.74 & $2572.52 \mathrm{a}$ & $1609.66 \mathrm{a}$ & $548.58 \mathrm{a}$ & $542.23 \mathrm{a}$ & $1.50 \mathrm{~b}$ & $1.91 \mathrm{a}$ \\
\hline 2nd Cutting & 42.02 & $11.85 \mathrm{a}$ & $8.27 \mathrm{a}$ & $13.19 \mathrm{~b}$ & $6682.32 \mathrm{~b}$ & 3090.03 & $1757.16 \mathrm{~b}$ & $990.58 \mathrm{~b}$ & $278.22 \mathrm{~b}$ & $413.33 \mathrm{~b}$ & $2.19 \mathrm{a}$ & $1.66 \mathrm{~b}$ \\
\hline $\operatorname{LSD}(\% 5)$ & ns & 0.84 & 0.91 & 2.82 & 983.50 & ns & 370.50 & 265.40 & 103.30 & 87.30 & 0.12 & 0.19 \\
\hline Overall mean & 42.95 & 9.48 & 7.58 & 17.51 & 7663.71 & 3130.38 & 2164.84 & 1300.12 & 413.40 & 477.78 & 1.85 & 1.78 \\
\hline
\end{tabular}

*Levels not connected by the same letter are significantly $(\mathrm{p}<0.05)$ different according to the Least Significant Difference test 
Dry herbage yield ranged from 1076.17 to 1418.96 with a mean of $1300.12 \mathrm{~kg} \mathrm{ha}^{-1}$ among the planting dates. Minimum dry herbage yield and dry leaves yield was observed with purple basil, while the maximum was recorded for cinnamon basil. Dry leaves yield ranged from 403.29 to 565.20 with a mean of $477.78 \mathrm{~kg} \mathrm{ha}^{-1}$ among the planting dates. Dry flowers yield ranged from 291.95 to 612.79 with a mean of $413.40 \mathrm{~kg} \mathrm{ha}^{-1}$ among the planting dates. Minimum dry flowers yield was obtained with purple basil, while the maximum was recorded for lemon basil. Vieira and Simon (2006) and Nurzynska-Wierdak (2007), similar to our findings, stated that lemon basil had higher values of dry herbage weight than purple basil. Chang et al. (2005) reported that at temperatures of $30^{\circ} \mathrm{C}$, the maximum dry matter content was obtained. This shows that the purple basil, which is very sensitive to environmental conditions, is highly affected even by short-term temperature fluctuations in climatic conditions. Since purple basil is a sensitive chemotype, its long adaptation caused the plant mass to be low and the spikes to be small with the delay in flowering, so dry flower weights have decreased.

While the cultivars were exposed to the same field environment, the essential oils differed in color, cloudiness, constituents and yield based on the plant organs. Leaves essential oil rate ranged from 1.78 to 1.89 with a mean of $1.85 \mathrm{ml} / 100 \mathrm{~g}$ among the planting dates. The minimum leaves and flowers essential oil rate were obtained with purple basil, while the maximum was observed for cinnamon basil. Flower essential oil rates ranged from 1.43 to 2.04 with a mean of $1.78 \mathrm{ml} / 100 \mathrm{~g}$. The essential oil rate in the previous studies was found different; for cinnamon basil 0.20 to $1.89 \mathrm{ml} / 100 \mathrm{~g}$ (Vieira and Simon, 2006; Nurzyńska-Wierdak, 2013; Tsasi et al., 2017), for purple basil 0.58 to $1.10 \mathrm{ml} / 100 \mathrm{~g}$ (Phippen and Simon, 2000; Vieira and Simon, 2006; Nurzynska-Wierdak, 2007; Ekren et al., 2012; Maggio et al., 2016), for lemon basil 0.36 to $1.18 \mathrm{ml} / 100 \mathrm{~g}$ (Tansi and Nacar, 2000; Vieira and Simon, 2006; Nurzynska-Wierdak, 2007, 2013). Our results were close or higher than these studies; with a mean of $2.18 \mathrm{ml} / 100 \mathrm{~g}$ and $2.61 \mathrm{ml} / 100 \mathrm{~g}$ for leaves and flowers of cinnamon basil, $1.04 \mathrm{ml} / 100 \mathrm{~g}$ and $1.55 \mathrm{ml} / 100 \mathrm{~g}$ for leaves and flowers of purple basil, $2.32 \mathrm{ml} / 100 \mathrm{~g}$ and $1.20 \mathrm{ml} / 100 \mathrm{~g}$ for leaves and flowers of lemon basil, respectively. The large differences in the content of basil essential oils in different geographic regions are due to different agricultural conditions and different agricultural cultivation techniques (Sullivan, 2009; Atghaei et al., 2015). Further, it is reasoned that the high temperature caused by climate change at the beginning of flowering may have increased the rate of essential oil. Especially in cinnamon and lemon basil, more leaf essential oil rate was obtained due to the hot weather in the 2nd cutting, but this was not reflected in the flower essential oil rate. Results of the present and previous studies confirm great morphological and developmental variability of basil.

\section{Chemical composition of essential oil}

The chemical composition of essential oils for different basil cultivars was analyzed by GC/MS, and obtained results were summarized (Tables 3 and 4). The chemical composition of essential oils varied according to planting times, cuttings, cultivars and organs. Representative GC-MS chromatograms of the cultivars were given in Fig. 3. Sixty-five components were found, representing 82.29-98.50\% of the total essential oil. Vieira and Simon (2000) have reported volatile oil profiles in cultivars of several different Ocimum species, further demonstrating the importance of genetics as well as the environment on essential oil composition. 
Table 3. Essential oil components of basil cultivars in 1st cutting

\begin{tabular}{|c|c|c|c|c|c|c|c|c|c|c|c|c|c|c|c|c|c|c|c|}
\hline \multirow{2}{*}{\multicolumn{2}{|c|}{$\begin{array}{c}\text { Cultivars } \\
\text { Planting Time }\end{array}$}} & \multicolumn{6}{|c|}{ Cinnamon Basil } & \multicolumn{6}{|c|}{ Purple Basil } & \multicolumn{6}{|c|}{ Lemon Basil } \\
\hline & & $\begin{array}{c}1 \\
\text { April }\end{array}$ & $\begin{array}{c}1 \\
\text { April }\end{array}$ & \begin{tabular}{|c|}
15 \\
April
\end{tabular} & $\begin{array}{c}15 \\
\text { April }\end{array}$ & $\begin{array}{c}30 \\
\text { April }\end{array}$ & $\begin{array}{c}30 \\
\text { April }\end{array}$ & $\begin{array}{c}1 \\
\text { April }\end{array}$ & $\begin{array}{c}1 \\
\text { April }\end{array}$ & \begin{tabular}{|c|}
15 \\
April \\
\end{tabular} & $\begin{array}{c}15 \\
\text { April }\end{array}$ & \begin{tabular}{|c|}
30 \\
April
\end{tabular} & $\begin{array}{c}30 \\
\text { April }\end{array}$ & $\begin{array}{c}1 \\
\text { April }\end{array}$ & $\begin{array}{c}1 \\
\text { April }\end{array}$ & $\begin{array}{c}15 \\
\text { April }\end{array}$ & $\begin{array}{c}15 \\
\text { April }\end{array}$ & \begin{tabular}{|c|}
30 \\
April \\
\end{tabular} & $\begin{array}{c}30 \\
\text { April } \\
\end{array}$ \\
\hline \multicolumn{2}{|l|}{ Organs } & Leaves & Flower: & Leaves & Flowers & Leaves & Flower & Leaves & Flower & Leaves & Flower & Leaves & Flower & Leaves & Flower & Leaves & Flower & Leaves & Flowers \\
\hline Components & $\begin{array}{c}\text { RT } \\
(\mathbf{m i n})\end{array}$ & \multicolumn{18}{|c|}{ Area (\%) } \\
\hline beta-Pinene & 9.401 & 0.30 & 0.14 & 0.25 & 0.08 & 0.28 & 0.07 & 1.30 & 0.90 & 1.53 & 0.83 & 1.68 & 0.77 & - & 0.08 & - & 0.08 & - & 0.15 \\
\hline Sabinene & 9.532 & 0.12 & 0.06 & - & 0.03 & 0.10 & 0.03 & 0.65 & 0.41 & 0.27 & - & 0.33 & - & - & - & - & - & - & 0.01 \\
\hline Santalone & 9.591 & - & - & - & - & - & - & - & - & - & - & - & - & - & - & - & - & - & - \\
\hline Myrcene & 9.597 & 0.21 & 0.11 & 0.34 & - & 0.20 & - & 1.49 & 0.61 & 2.49 & 1.10 & 2.94 & 1.03 & - & - & - & - & 0.07 & - \\
\hline Limonene & 10.048 & 0.29 & 0.20 & 0.26 & 0.16 & 0.34 & 0.16 & 0.57 & 0.25 & 0.72 & 0.26 & 0.91 & 0.27 & - & 0.10 & 0.14 & 0.10 & 0.13 & 0.15 \\
\hline beta-Ocimene & 10.303 & - & - & 0.03 & - & 0.03 & - & - & - & - & - & - & - & 0.18 & - & - & - & - & - \\
\hline beta-Phellandrene & 10.488 & - & - & - & - & - & - & - & - & - & - & - & - & - & - & - & - & - & - \\
\hline 3-Carene & 10.582 & 0.40 & 0.57 & 0.82 & 0.40 & 0.89 & 0.41 & - & - & - & - & - & - & - & 0.24 & 0.29 & 0.27 & 0.31 & - \\
\hline gamma-Terpinene & 10.856 & - & - & - & - & - & - & - & 0.01 & - & - & - & - & - & - & - & - & - & 0.15 \\
\hline Terpinolene & 10.903 & - & 0.05 & 0.05 & 0.05 & 0.05 & 0.05 & - & - & - & - & - & - & - & - & - & - & - & - \\
\hline Eucalyptol & 11.034 & 4.76 & 2.40 & 4.11 & 1.87 & 4.49 & 1.70 & 0.63 & 9.97 & 16.85 & 9.25 & 17.61 & 8.67 & - & 0.12 & 0.13 & 0.33 & 0.19 & 0.18 \\
\hline P-Cymene & 11.420 & 0.51 & 0.22 & 0.37 & 0.17 & 0.42 & 0.13 & - & - & - & - & - & - & - & - & - & - & - & - \\
\hline Hexyl acetate & 11.526 & - & - & - & - & - & - & - & - & - & - & - & - & - & 0.05 & - & - & - & 0.10 \\
\hline cis-3-Hexenyl acetate & 12.137 & - & - & - & - & - & - & - & - & - & - & - & - & - & 0.18 & 0.06 & 0.13 & 0.05 & 0.08 \\
\hline 2-Hexenal & 12.221 & 0.03 & - & 0.04 & - & - & - & - & - & - & - & - & - & - & - & - & - & - & - \\
\hline Octanal & 12.476 & - & - & - & - & - & - & - & - & - & - & - & - & - & 0.25 & - & 0.11 & - & 0.88 \\
\hline Rosefuran & 12.585 & - & - & - & - & - & - & - & - & - & - & - & - & 0.12 & 0.27 & 0.25 & 0.11 & 0.23 & - \\
\hline Octenyl acetate & 12.612 & - & - & - & - & - & - & 0.08 & - & - & 0.06 & - & - & - & - & - & - & - & - \\
\hline alpha-Cubebene & 12.684 & 0.05 & 0.05 & 0.17 & 0.06 & - & 0.06 & - & - & 0.05 & 0.07 & - & - & - & - & - & - & - & - \\
\hline Perillene & 12.927 & - & - & - & - & - & - & - & - & - & - & - & - & - & - & 0.06 & 0.07 & 0.06 & 0.06 \\
\hline Sulcatone & 13.263 & - & - & - & - & - & - & - & - & - & - & - & - & 0.57 & 1.02 & 1.21 & 1.02 & 0.93 & 1.08 \\
\hline Copaene & 13.265 & 0.11 & 0.17 & - & 0.28 & 0.10 & 0.24 & 0.29 & - & 0.22 & 0.35 & - & 0.35 & - & - & - & - & - & - \\
\hline beta-Bourbonene & 13.829 & 0.09 & 0.05 & - & 0.09 & 0.11 & 0.13 & - & - & 0.11 & 0.13 & 0.11 & 0.23 & - & - & - & - & - & - \\
\hline Octyl acetate & 13.906 & - & - & - & - & - & - & 0.60 & - & - & - & - & - & - & 0.78 & - & 0.74 & - & 1.87 \\
\hline Linalooloxide (cis, isomer B) & 13.966 & 0.48 & 0.53 & 0.49 & 0.47 & 0.29 & 0.36 & - & - & 0.22 & - & 0.13 & - & - & - & 0.49 & - & 0.55 & - \\
\hline
\end{tabular}


Table 3. (continued)

\begin{tabular}{|c|c|c|c|c|c|c|c|c|c|c|c|c|c|c|c|c|c|c|c|}
\hline \multirow{2}{*}{\multicolumn{2}{|c|}{$\begin{array}{c}\text { Cultivars } \\
\text { Planting Time }\end{array}$}} & \multicolumn{6}{|c|}{ Cinnamon Basil } & \multicolumn{6}{|c|}{ Purple Basil } & \multicolumn{6}{|c|}{ Lemon Basil } \\
\hline & & $\begin{array}{c}1 \\
\text { April }\end{array}$ & $\begin{array}{c}1 \\
\text { April }\end{array}$ & \begin{tabular}{|c|}
15 \\
April
\end{tabular} & $\begin{array}{c}15 \\
\text { April }\end{array}$ & $\begin{array}{c}30 \\
\text { April }\end{array}$ & $\begin{array}{c}30 \\
\text { April }\end{array}$ & $\begin{array}{c}1 \\
\text { April }\end{array}$ & $\begin{array}{c}1 \\
\text { April }\end{array}$ & $\begin{array}{c}15 \\
\text { April }\end{array}$ & $\begin{array}{c}15 \\
\text { April }\end{array}$ & $\begin{array}{c}30 \\
\text { April } \\
\end{array}$ & $\begin{array}{c}30 \\
\text { April }\end{array}$ & $\begin{array}{c}1 \\
\text { April }\end{array}$ & $\begin{array}{c}1 \\
\text { April }\end{array}$ & $\begin{array}{c}15 \\
\text { April }\end{array}$ & $\begin{array}{c}15 \\
\text { April }\end{array}$ & \begin{tabular}{|c|}
30 \\
April
\end{tabular} & $\begin{array}{c}\text { 30 } \\
\text { April }\end{array}$ \\
\hline \multicolumn{2}{|l|}{ Organs } & \multicolumn{18}{|c|}{ LeavesFlowersLeavesFlowersLeaves FlowersLeavesFlowersLeaves FlowersLeavesFlowers Leaves Flowers LeavesFlowersLeavesFlowers } \\
\hline Components & RT (min) & \multicolumn{18}{|c|}{ Area $(\%)$} \\
\hline Sabinene hydrate & 14.209 & 0.33 & 0.30 & 0.32 & 0.46 & - & 0.37 & - & - & 0.27 & - & 0.30 & 0.33 & - & - & - & - & - & - \\
\hline (+)-cis-alpha-Bergamotene & 14.239 & 0.53 & - & 0.65 & 0.57 & 0.82 & - & 1.65 & - & 1.12 & 2.10 & 0.83 & 1.76 & 0.47 & 1.45 & 0.74 & 1.46 & 0.77 & 2.23 \\
\hline Exo-Fenchyl acetate & 14.429 & - & - & - & - & - & - & 1.05 & - & - & - & 1.35 & - & 0.62 & - & - & - & - & - \\
\hline Linalool & 14.506 & 24.87 & 22.68 & 24.20 & 21.48 & 18.05 & 14.46 & 46.47 & 39.19 & 44.20 & 38.01 & 40.53 & 37.33 & 2.88 & 7.11 & 5.15 & 7.27 & 4.81 & 7.50 \\
\hline beta-Elemene & 14.809 & - & - & - & 10.38 & - & 6.77 & - & 16.91 & - & 17.69 & - & 17.53 & - & - & - & - & - & - \\
\hline Myroxide & 14.945 & - & - & - & - & - & - & - & - & - & - & - & - & - & 0.92 & - & - & - & - \\
\hline beta-Caryophyllene & 15.404 & 0.29 & - & - & 0.79 & 0.21 & 0.66 & 1.48 & 2.18 & 1.72 & 2.39 & 2.04 & 2.54 & 0.74 & 2.05 & 0.84 & 1.64 & 0.93 & 2.65 \\
\hline alpha-Bisabolene & 15.776 & - & - & 0.27 & - & - & - & - & 0.54 & - & 0.44 & - & 0.50 & - & - & - & - & - & 1.80 \\
\hline Chrysanthenol & 15.791 & - & - & - & - & - & - & - & - & - & - & - & - & 2.84 & - & - & 2.31 & 3.31 & - \\
\hline Guaiyl acetate & 15.972 & - & - & - & - & - & - & - & 2.78 & - & 3.22 & - & 3.46 & - & - & - & - & - & - \\
\hline gamma-Gurjunene & 15.972 & - & - & - & - & - & - & 1.48 & - & 1.91 & - & 2.10 & - & - & - & - & - & - & - \\
\hline delta-Guaiene & 15.990 & - & 2.87 & - & 3.66 & - & - & - & - & - & - & - & - & - & - & - & - & - & - \\
\hline Terpinen-4-ol & 16.002 & 2.14 & - & 2.31 & - & 2.76 & - & - & - & - & - & - & - & - & - & - & - & - & - \\
\hline trans-Chrysanthemal & 16.055 & - & - & - & - & - & - & - & - & - & - & - & - & 0.96 & - & 1.25 & - & 1.57 & - \\
\hline beta-Bisabolene & 16.123 & - & - & - & - & - & - & - & - & - & - & - & - & 1.85 & 4.65 & 1.82 & 3.16 & 2.07 & 4.82 \\
\hline Humulene & 16.357 & - & 0.74 & 0.52 & 1.26 & - & 1.24 & 0.53 & 1.09 & 0.52 & 1.17 & 0.71 & 1.36 & - & - & - & - & - & - \\
\hline gamma-Cadinene & 16.470 & 1.40 & 1.34 & 1.39 & 1.85 & 1.48 & 1.24 & 1.40 & 1.38 & 1.46 & 1.62 & 1.78 & 1.78 & - & - & - & - & - & - \\
\hline Germacrene D & 16.731 & 0.48 & 1.97 & - & 3.34 & 0.59 & 2.75 & 0.94 & 3.69 & 1.51 & 4.68 & 1.93 & 4.95 & - & 0.92 & 0.34 & 0.71 & - & 0.68 \\
\hline Nerol & 16.829 & - & - & - & - & - & - & - & - & - & - & - & - & 0.54 & 0.96 & 0.63 & 0.87 & 1.03 & 1.59 \\
\hline Estragole & 16.880 & 5.39 & 5.52 & 25.50 & 6.77 & 3.19 & 3.76 & - & - & - & - & - & - & - & - & - & - & - & - \\
\hline alpha-Terpineol & 17.034 & - & - & - & - & - & - & 3.18 & 3.52 & 3.98 & 1.67 & 4.45 & 1.66 & 0.81 & 2.18 & 1.08 & 1.14 & 1.51 & 1.57 \\
\hline Camphene & 17.123 & - & - & - & - & - & - & - & - & - & 1.78 & - & 2.24 & 0.82 & - & - & - & - & - \\
\hline Isoborneol (Isomer 2) & 17.420 & 0.26 & 0.42 & - & 0.43 & 0.27 & 0.46 & - & - & - & - & - & - & - & - & - & - & - & - \\
\hline Geraniol & 17.717 & - & - & - & 0.39 & - & - & 0.51 & 0.31 & 0.73 & 0.49 & 0.94 & 0.64 & 1.89 & 6.13 & 3.82 & 8.63 & 3.34 & 3.85 \\
\hline Calamenene & 18.174 & 0.16 & 0.18 & 0.16 & - & 0.20 & 0.20 & - & - & - & - & - & - & - & - & - & - & - & - \\
\hline
\end{tabular}


Table 3. (continued)

\begin{tabular}{|c|c|c|c|c|c|c|c|c|c|c|c|c|c|c|c|c|c|c|c|}
\hline \multirow{2}{*}{\multicolumn{2}{|c|}{$\begin{array}{c}\text { Cultivars } \\
\text { Planting Time }\end{array}$}} & \multicolumn{6}{|c|}{ Cinnamon Basil } & \multicolumn{6}{|c|}{ Purple Basil } & \multicolumn{6}{|c|}{ Lemon Basil } \\
\hline & & $\begin{array}{c}1 \\
\text { April }\end{array}$ & $\begin{array}{c}1 \\
\text { April }\end{array}$ & \begin{tabular}{|c|}
15 \\
April \\
\end{tabular} & $\begin{array}{c}15 \\
\text { April }\end{array}$ & $\begin{array}{c}30 \\
\text { April }\end{array}$ & $\begin{array}{c}30 \\
\text { April }\end{array}$ & $\begin{array}{c}1 \\
\text { April } \\
\end{array}$ & $\begin{array}{c}1 \\
\text { April }\end{array}$ & $\begin{array}{c}15 \\
\text { April } \\
\end{array}$ & $\begin{array}{c}15 \\
\text { April }\end{array}$ & $\begin{array}{c}\mathbf{3 0} \\
\text { April } \\
\end{array}$ & $\begin{array}{c}30 \\
\text { April } \\
\end{array}$ & $\begin{array}{c}1 \\
\text { April } \\
\end{array}$ & $\begin{array}{c}1 \\
\text { April }\end{array}$ & \begin{tabular}{|c|}
15 \\
April \\
\end{tabular} & $\begin{array}{c}15 \\
\text { April } \\
\end{array}$ & $\begin{array}{c}30 \\
\text { April } \\
\end{array}$ & $\begin{array}{c}30 \\
\text { April } \\
\end{array}$ \\
\hline \multicolumn{2}{|l|}{ Organs } & Leaves & Flowers & Leaves & Flowers & Leaves & Flowers & Leaves & Flowers & Leaves & Flower & Leaves & Flowers & Leaves & Flower & Leaves & Flowers & Leaves & Flowers \\
\hline Components & $\begin{array}{c}\text { RT } \\
(\mathbf{m i n})\end{array}$ & \multicolumn{18}{|c|}{ Area $(\%)$} \\
\hline Neral & 18.373 & - & - & 0.09 & - & - & - & - & 0.44 & - & - & - & - & 33.36 & 26.41 & 33.27 & 22.52 & 29.37 & 22.10 \\
\hline Citral & .951 & 0.13 & - & 0.08 & - & - & - & 0.29 & 0.40 & 0.55 & 0.07 & - & - & 44.43 & 33.53 & 38.25 & 26.26 & 37.71 & 30.20 \\
\hline Nerolidol & 19.741 & 0.25 & 0.94 & 0.75 & 1.60 & 0.79 & 1.76 & - & 0.52 & 0.33 & 0.51 & 0.41 & 0.54 & - & - & - & - & - & - \\
\hline trans-Muurola-4(14),5-diene & 20.370 & 0.04 & - & 0.02 & 0.15 & 0.11 & 0.12 & 0.05 & 0.09 & 0.07 & 0.10 & 0.10 & - & - & - & - & - & - & - \\
\hline p-Mentha-3,8-diene & 20.566 & - & - & - & - & - & - & - & - & - & - & - & - & 0.10 & 0.25 & 0.13 & 0.42 & 0.25 & 0.58 \\
\hline Di-epi-1,10-cubenol & 20.880 & 0.38 & 0.46 & 0.52 & 0.59 & 0.48 & 0.58 & 0.60 & 0.53 & 0.49 & 0.57 & 0.53 & 0.62 & - & - & - & - & - & - \\
\hline Methyl Eugenol & 21.290 & - & - & - & - & - & - & 0.50 & - & 0.44 & - & 1.15 & - & - & - & - & - & - & - \\
\hline tau-Cadinol & 21.895 & 2.19 & 2.70 & 4.36 & 3.42 & 2.96 & 4.89 & 2.45 & 4.64 & 2.55 & 4.81 & 2.85 & 5.26 & - & - & - & - & - & - \\
\hline Caryophyllene oxide & 22.021 & - & - & - & - & - & - & - & - & - & - & - & - & 1.97 & 2.75 & 1.90 & 3.04 & 2.92 & 4.61 \\
\hline gamma-Muurolene & 22.056 & 0.82 & 0.97 & - & 1.39 & 1.08 & - & 1.88 & - & 1.51 & 0.59 & 1.32 & - & - & - & - & - & - & - \\
\hline Methyl cinnamate & 22.631 & 37.36 & 36.08 & 22.03 & 31.02 & 40.16 & 44.08 & - & - & - & - & - & - & - & - & - & - & - & - \\
\hline Humulene epoxide II & 22.643 & - & - & - & - & - & - & - & - & - & - & - & - & - & 0.23 & 0.65 & - & 0.72 & 1.18 \\
\hline alpha-Cadinol & 22.725 & - & - & - & - & - & - & 0.67 & 0.29 & 0.19 & 0.29 & 0.23 & 0.41 & 0.93 & 0.41 & - & 0.38 & - & - \\
\hline Eugenol & 23.088 & 2.27 & 3.26 & 1.31 & 1.80 & 1.84 & - & 13.70 & 5.41 & 10.01 & 4.07 & 9.27 & 2.83 & - & 0.21 & 0.31 & 0.54 & 0.26 & 0.42 \\
\hline beta-Eudesmol & 23.456 & - & - & 0.15 & - & - & 0.39 & - & - & - & - & - & 0.44 & - & - & - & - & - & - \\
\hline & Total & 86.64 & 84.98 & 91.56 & 95.01 & 82.29 & 87.07 & 84.44 & 96.06 & 96.02 & 98.32 & 96.53 & 97.50 & 96.08 & 93.25 & 92.81 & 83.31 & 93.09 & 90.49 \\
\hline
\end{tabular}


Table 4. Essential oil components of basil cultivars in 2nd cutting

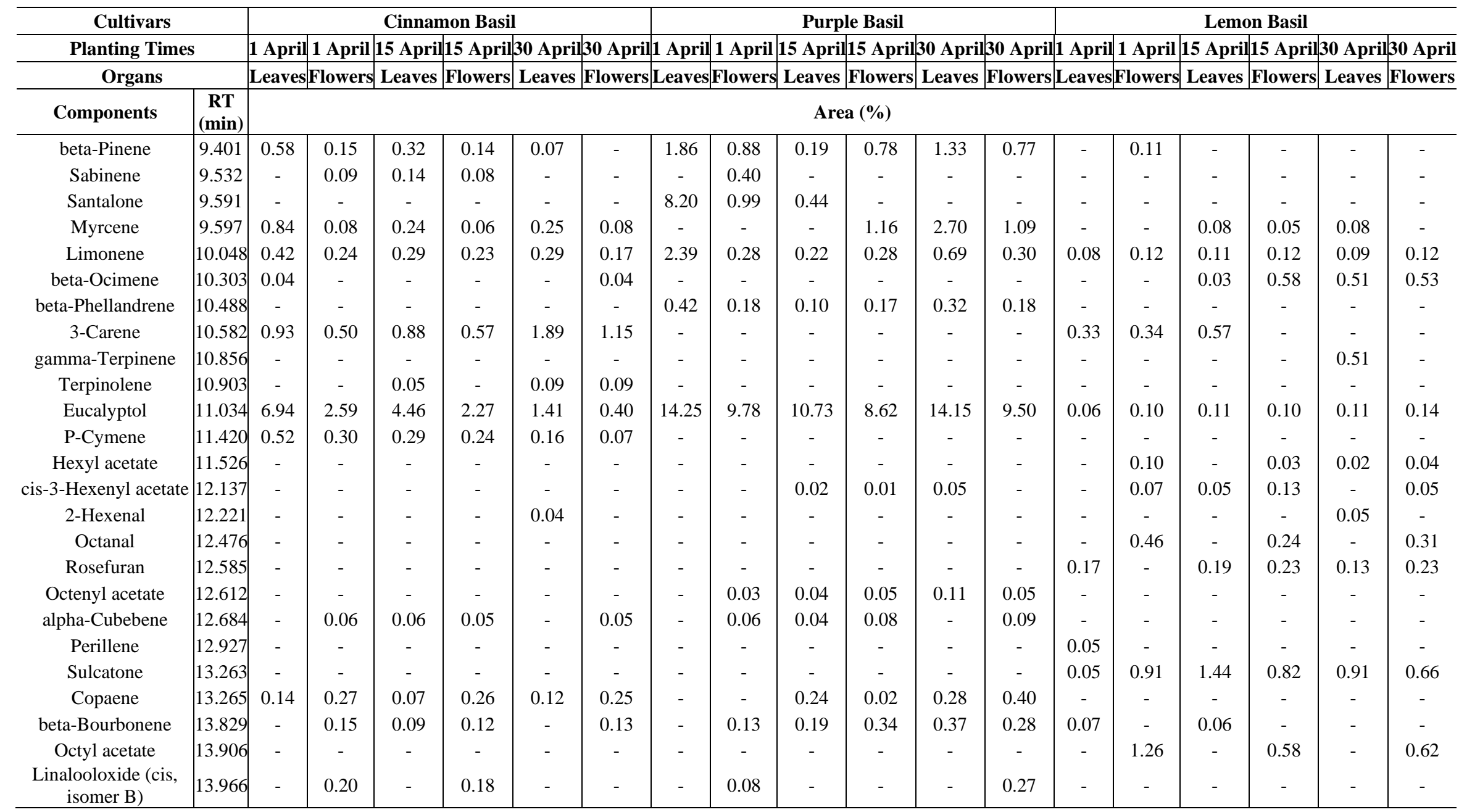


Table 4. (continued)

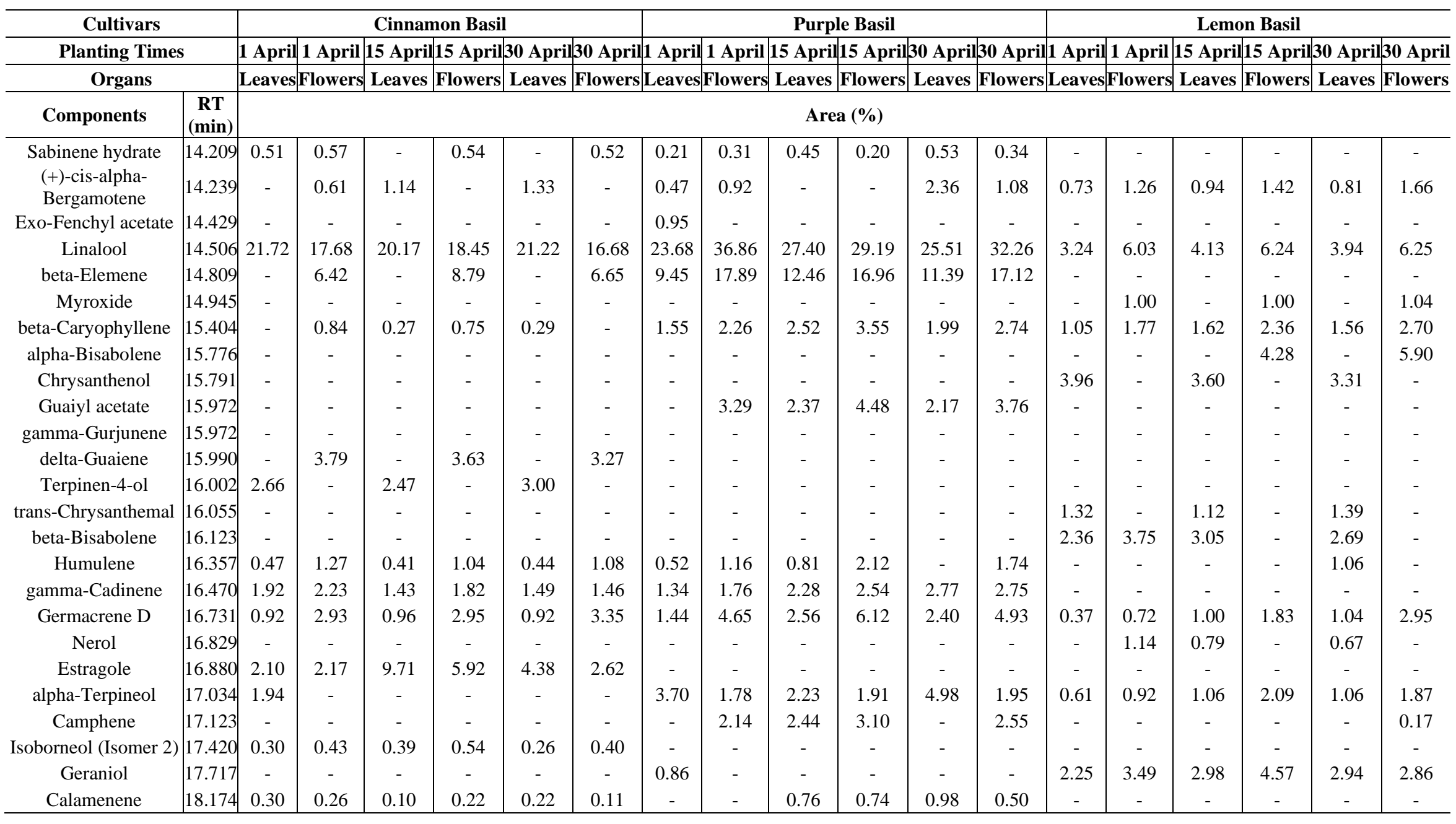


Table 4. (continued)

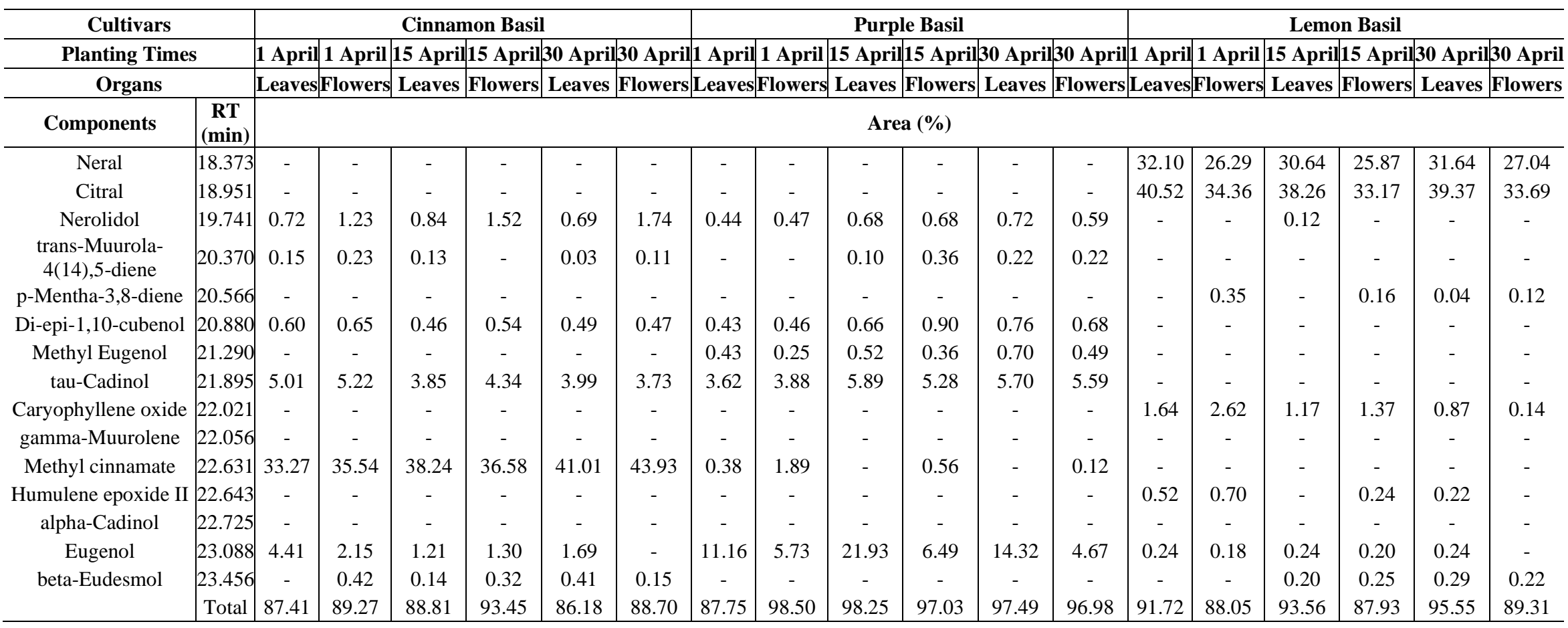




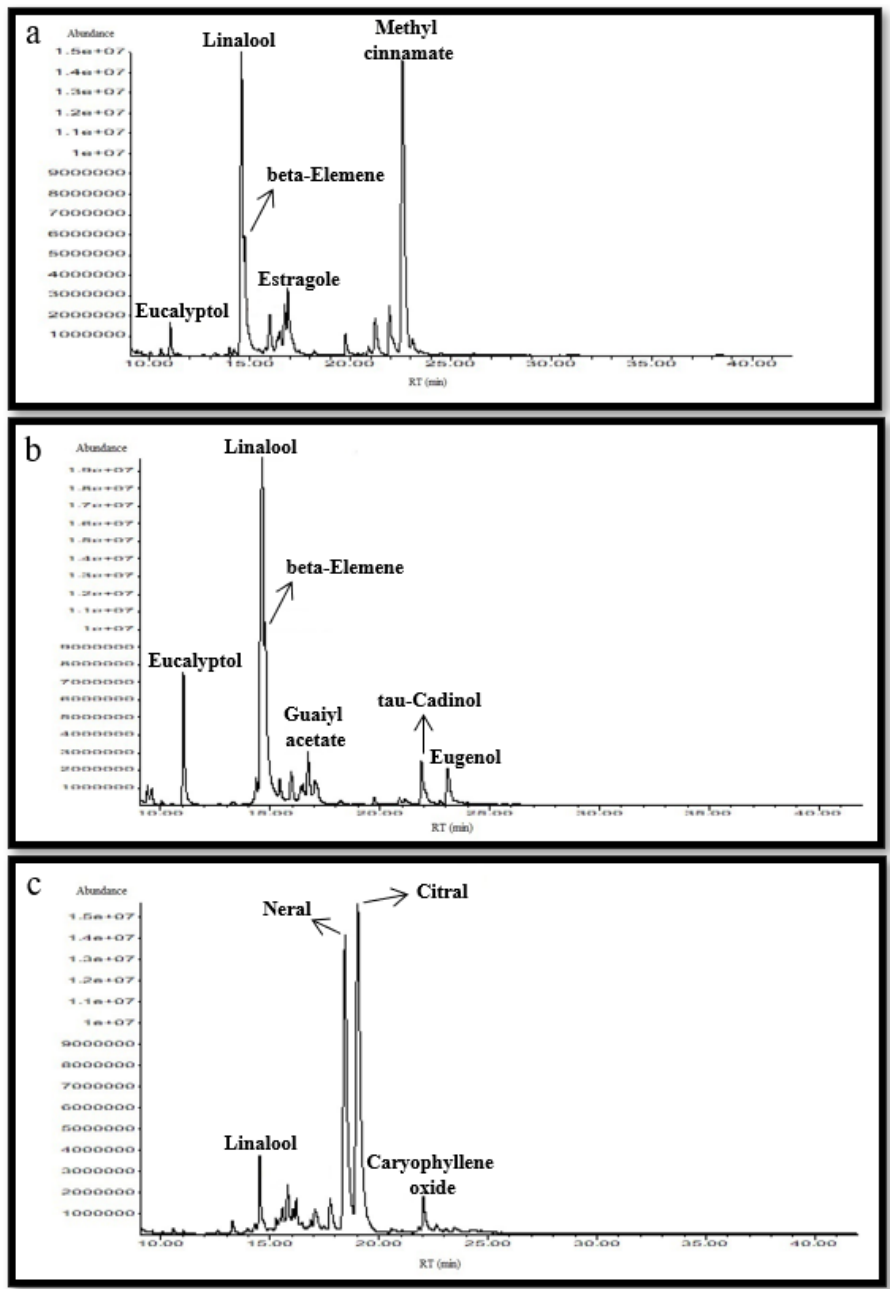

Figure 3. The representative GC/MS chromatogram of different basil cultivars a: cinnamon basil, b: purple basil, c: lemon basil

The major components for cinnamon basil were methyl cinnamate (22.03-44.08\%), linalool (14.46-24.87\%), estragole (2.10-9.71\%) and eucalyptol (0.40-6.94\%), respectively. For methyl cinnamate, the highest values were obtained for both flower and leaf in 30 April planting time. Many of the studies reported that methyl cinnamate was found as a major component in essential oils of cinnamon basil (Schulz et al., 2003; Viña and Murillo, 2003; Nurzyńska-Wierdak, 2013; da Costa et al., 2014). Padalia et al. (2017) report that methyl cinnamate accumulates more in cold temperatures. On the contrary, although hot weather prevails in our region, methyl cinnamate rate was found to be similar.

The major components for purple basil were linalool (23.68-46.47\%), eugenol (2.83-21.93\%), beta-elemene (9.45-17.89\%) and eucalyptol (0.63-17.61\%), respectively. In the previous studies, linalool was obtained as a major component in essential oils of purple basil by Nacar and Tansi (2000), Mondello et al. (2002), Vieira et al. (2014), Varga et al. (2017), Ahmed et al. (2017). Gill and Randhawa (1992) observed that a delay in planting time increased the linalool content and decreased the methyl chavicol and eugenol content. Nevertheless, in our study linalool content decreased based on a delay in planting time for cinnamon and lemon basil. Linalool was found more in purple basil 
leaves than in flowers and reduced in the 2nd cutting compared to the 1st cutting for all planting times. This may be related to the slower growth of purple basil in the 2nd cuttings. In contrast to Carvalho Filho et al. (2006), we got more linalool content in the leaves than the flowers in 1st cuttings. However, this situation changed with the 2nd cutting; the flowers contained more linalool with the effect of increasing temperature.

The major components for lemon basil were citral (26.26-44.43\%), neral (22.10-33.36\%), geraniol (1.89-8.63\%) and linalool (2.88-7.50\%), respectively. In both cuttings, the highest citral rate was detected at the 1 April planting time in lemon basil. Citral was also found as a major component in essential oils of lemon basil in other studies investigated by Tansi and Nacar (2000), Al-Kateb and Mottram (2014), Dombrov et al. (2019). Simon et al. (1990) and Morales and Simon (1996) reported a strong lemony flavor and high citral content in lemon basil. On the contrary to Al-Kateb and Mottram (2014), it was found that citral and neral were found to be higher in the leaves of lemon basil compared to flowers in both planting and cuttings times.

Limonene, eucalyptol, (+)-cis-alpha-bergamotene, linalool, germacrene D and eugenol were the components that were observed almost for all planting and cutting times, cultivars and leaves and flowers. Santalone and beta-phellandrene were only observed 2nd cutting for purple basil, as gamma-gurjunene was only found 1st cutting in purple basil leaves. Methyl eugenol was observed 1st cutting in purple basil leaves, while 2nd cutting was found both leaves and flowers for all planting times. Trans-chrysanthemal was observed both 1 st cutting and 2nd cutting in leaves of lemon basil for all planting times, while octyl acetate was only found in lemon basil flowers. Delta-guaiene was found both 1st cutting and 2nd cutting in flowers of cinnamon basil, while Terpinen-4-ol was obtained both 1 st cutting and 2 nd cutting in leaves of cinnamon basil. Alpha-cadinol was only observed 1st cutting in purple and lemon basil for all planting times. The highest estragole content $(25.20 \%)$ was determined in cinnamon basil flowers at the 1 st cutting time, while maximum methyl cinnamate content $(44.08 \%)$ was observed in cinnamon basil leaves at the 1st cutting time. The highest eucalyptol (17.61\%) and linalool (46.47\%) content was obtained in purple basil leaves at the 1st cutting, while maximum santalone (8.20\%), tau-cadinol (5.89\%), eugenol content (21.93\%) was found in purple basil leaves at the 2nd cutting. Eugenol was found more in leaves of purple basil than in flowers for all planting times. Beta-elemene was found in purple basil in flowers and was not found in the leaves at the 1 st cutting time. Moreover, beta-elemene was found in purple basil more than leaves in flowers at the 2 nd cutting time for all planting time. The highest betaelemene $(17.89 \%)$ and germacrene D content $(6.12 \%)$ was determined in purple basil flowers at the 2nd cutting for all planting times. Maximum alpha-bisabolene content $(5.90 \%)$ was indicated in lemon basil flowers at the 2nd cutting time.

\section{Principal component analysis biplot of the treatments according to the important traits and main essential oil components}

Principal component analysis biplot (PCAbiplot) is used to express a data source with a few components that most reflect the variance of the data. It allows to shrink the data and reveals the connections among the variables that make up the data. Moreover, this analysis allows us to see positive and negative correlations. There is a positive correlation between the lines that are in the same direction and close to each other, while there is a negative correlation between the opposite and distant lines. PCAbiplot on correlations was performed to visualize the effect of different planting times, cultivars and cuttings on important traits and main essential oil components (Fig. 4). The experimental groups were 
separately discriminated with principal component analysis on correlations. For example, the positive correlation between citral, neral and flower length can be easily observed with the help of PCAbiplot analysis in the area where lemon basil was located. In terms of the important traits and essential oil components, clear discrimination was revealed on the plotted scores, where component 1 and component 2 accounted for $84.80 \%$ of the total variance. The first axis and second axis explained $61.20 \%$ and $23.60 \%$ of the total variance, respectively.

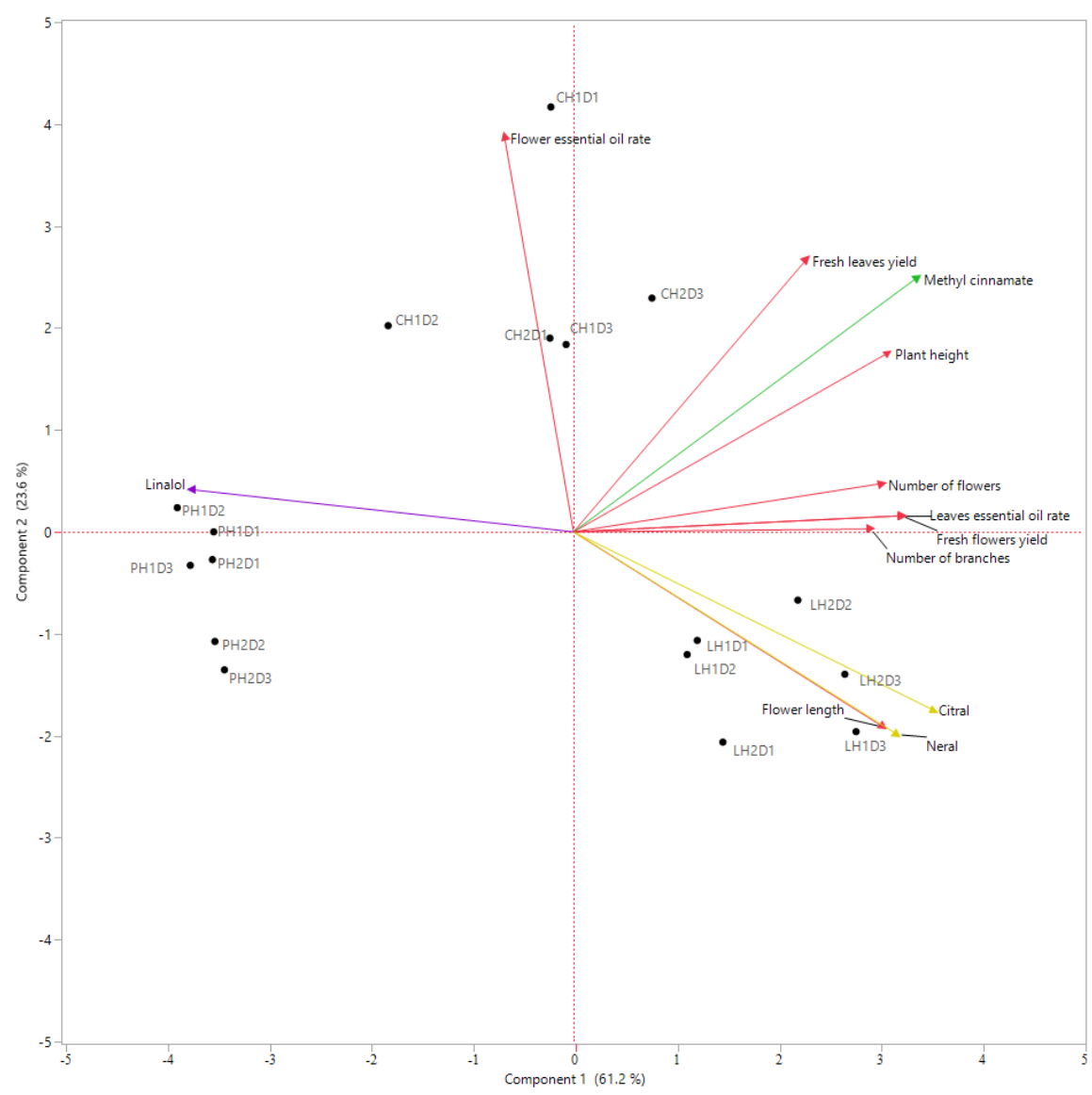

Figure 4. Principal component analysis on correlations of important traits and main essential oil components (C: Cinnamon Basil, P: Purple Basil, L: Lemon Basil; H1: $1^{\text {st }}$ Cutting, H2:2 Cutting; D1: 1 April Planting Time, D2: 15 April Planting Time, D3: 30 April Planting Time)

\section{Heat map for the main essential oil components corresponding to the treatments}

The changes in main essential oil components were structured using a heat map. According to the heat map (Fig. 5), the main essential oil components are visualized under planting times, cuttings, cultivars and organs. The differences between treatments can be observed easily with the help of the heat map. For instance; highest linalool content was noted in 1st cutting at 1 April planting time with purple basil leaves, highest citral content was noted in 1st cutting at 1 April planting time with lemon basil leaves and highest methyl cinnamate content was noted in 1st cutting at 30 April planting time with cinnamon basil flowers. Moreover, the differences can be observed clearly between leaves and flowers in the main components such as eucalyptol, linalool, citral, taucadinol, eugenol, neral and beta-elemene. 


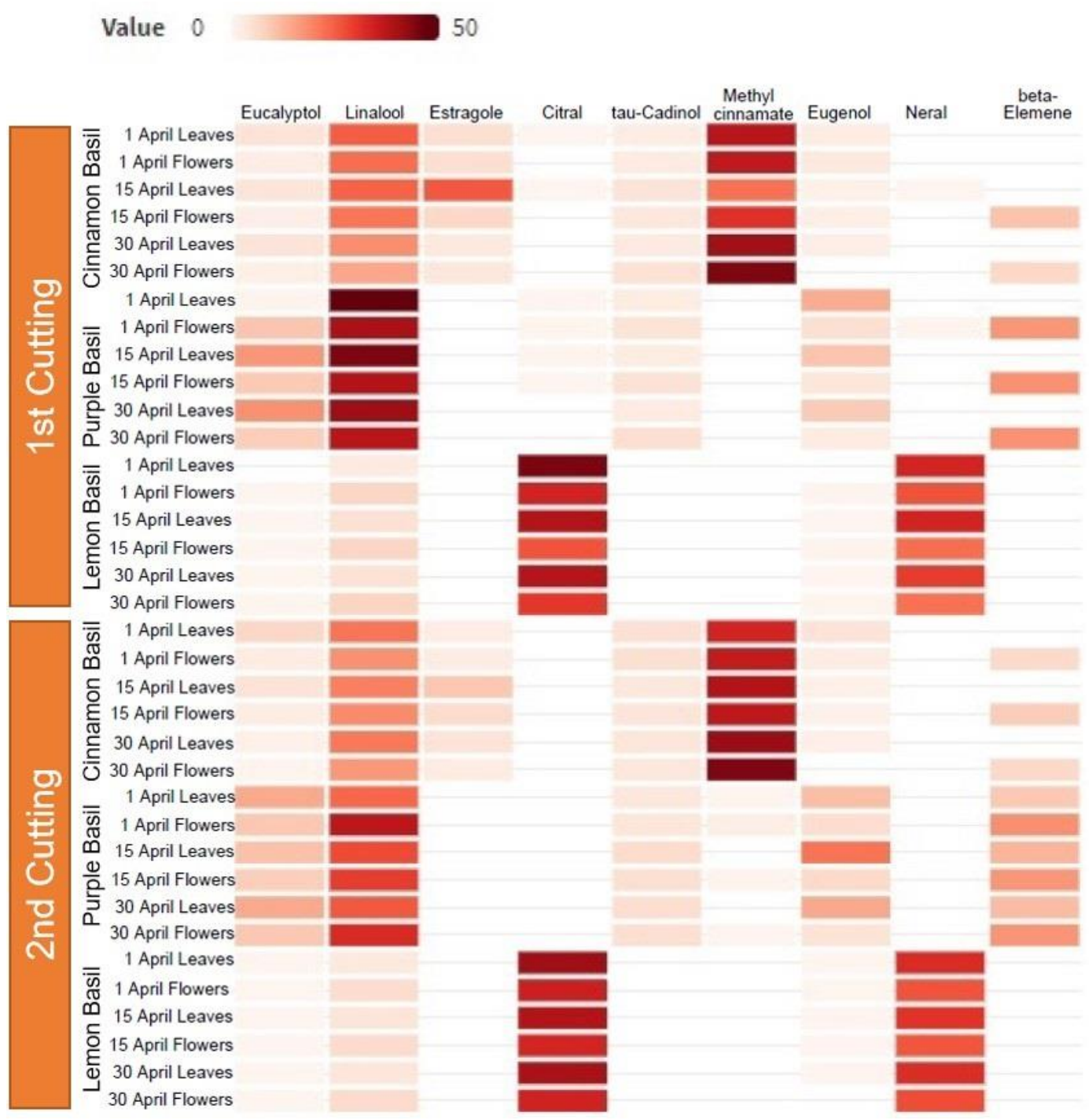

Figure 5. Heatmap for the main components of essential oils (Value=\%)

\section{Conclusions}

The current study comprehensively explored a wide range of quantitative trait variations in basil cultivars under hot Mediterranean environment, and it was found that planting times affects widely the agronomic traits and chemical composition of basil cultivars. However, high-temperature fluctuations occurring during the development period adversely affected the growth mechanism of the basil. Although more than two cuttings can be made depending on the region where basil is grown, in our region where global warming is felt more clearly and in parallel with the temperature increases, it did not show rapid growth. Since the leaves of basil are generally used, 1 April planting date can be recommended in terms of cultivation in this region. Purple basil has been found as a more sensitive cultivar compared to environmental conditions and has not shown a high performance under the conditions of our region. As a result of the effects of high temperatures observed in our region in May $\left(+40{ }^{\circ} \mathrm{C}\right)$, June-August $\left(+36{ }^{\circ} \mathrm{C}\right)$, while the essential oil rate in cinnamon and lemon basil increased, purple basil had a negative effect on plant growth rather than a serious increase in the rate of essential oil for all planting 
times. It can be suggested that purple basil should be grown in cool regions because of weakness to high temperatures. The essential oil of the studied basil cultivars can be used as a good source of chemical products and industrially important flavor components such as methyl cinnamate, linalool, citral and neral, which could be further improved by cultivar and agrotechnological research strategies. In further studies, it is recommended to carry out adaptation experiments of purple basil cultivars adapted to diverse stress environments due to changing essential oil components and yield traits.

Acknowledgements. This study was financially supported by the Scientific Research Project Unit of the Cukurova University (project No. FYL-2019-12296), Adana, Turkey.

\section{REFERENCES}

[1] Ahmed, A. H., Hussein, K., Alsyari, A. H. (2017): Chemotaxonomy and spectral analysis (GC/MS and FT-IR) of essential oil composition of two Ocimum basilicum L. varieties and their morphological characterization. - Jordan Journal of Chemistry 12(3): 147-160.

[2] Al-Huqail, A., El-Dakak, R. M., Sanad, M. N., Badr, R. H., Ibrahim, M. M., Soliman, D., Khan, F. (2020): Effects of climate temperature and water stress on plant Growth and accumulation of antioxidant compounds in Sweet Basil (Ocimum basilicum L.) leafy vegetable. - Scientifica 2020: 3808909.

[3] Al-Kateb, H., Mottram, D. S. (2014): The relationship between growth stages and aroma composition of lemon basil Ocimum citriodorum Vis. - Food Chemistry 152: 440-446.

[4] Applequist, W. L., Brinckmann, J. A., Cunningham, A. B., Hart, R. E., Heinrich, M., Katerere, D. R., Van Andel, T. (2020): Scientists' warning on climate change and medicinal plants. - Planta medica 86(1): 10-18.

[5] Atghaei, M., Rajabi, A., Hemayaty, S. (2015): Investigating the effect of bush density on the yield and leaf essence of green basil landraces (Ocimum basilicum). - International Journal of Research Studies in Agricultural Sciences 1: 11-17.

[6] Beillouin, D., Schauberger, B., Bastos, A., Ciais, P., Makowski, D. (2020): Impact of extreme weather conditions on European crop production in 2018. - Philosophical Transactions of the Royal Society B 375(1810): 20190510.

[7] Cakmakci, R., Milton, A. H. (2019): Effect of inoculation with plant-growth promoting rhizobacteria on development root systems of lemon basil (Ocimum x citriodorum Vis.). ICOFAAS 2019: 329.

[8] Carović-Stanko, K., Orlić, S., Politeo, O., Strikić, F., Kolak, I., Milos, M., Satovic, Z. (2010): Composition and antibacterial activities of essential oils of seven Ocimum taxa. Food Chemistry 119(1): 196-201.

[9] Carvalho Filho, J. L. S., Blank, A. F., Alves, P. B., Ehlert, P. A., Melo, A. S., Cavalcanti, S. C., de Fátima Arrigoni-Blank, M., Silva-Mann, R. (2006): Influence of the harvesting time, temperature and drying period on basil (Ocimum basilicum L.) essential oil. - Revista Brasileira de Farmacognosia 16(1): 24-30.

[10] Chang, X., Alderson, P., Wright, C. (2005): Effect of temperature integration on the growth and volatile oil content of basil (Ocimum basilicum L.). - The Journal of Horticultural Science and Biotechnology 80(5): 593-598.

[11] Chen, H., Jiang, J.-G. (2010): Osmotic adjustment and plant adaptation to environmental changes related to drought and salinity. - Environmental Reviews 18(NA): 309-319.

[12] da Costa, A. S., de Fátima Arrigoni-Blank, M., da Silva, M. A. A. P., Alves, M. F., de Alexandria Santos, D., Alves, P. B., Blank, A. F. (2014): The impact of hybridization on the volatile and sensorial profile of Ocimum basilicum L. - The Scientific World Journal 2014: 824594 . 
[13] De Masi, L., Siviero, P., Esposito, C., Castaldo, D., Siano, F., Laratta, B. (2006): Assessment of agronomic, chemical and genetic variability in common basil (Ocimum basilicum L.). - European Food Research and Technology 223(2): 273-281.

[14] Dombrov, L., Gille, E., Colţun, M., Necula, R. (2019): The composition of the essential oil of Ocimum basilicum var. citriodorum cultivated in the republic of Moldova. Рекомендовано до друку Науково-технічною радою Дослідної станції «Маяк» Інституту овочівництва і баштанництва НААН, протокол № 1 від 27 лютого 2019 р. Відповідальний за випуск: мол. наук. співроб. Позняк ОВ, 2, 127.

[15] Ekren, S., Sönmez, Ç., Özçakal, E., Kurttaş, Y. S. K., Bayram, E., Gürgülü, H. (2012): The effect of different irrigation water levels on yield and quality characteristics of purple basil (Ocimum basilicum L.). - Agricultural Water Management 109: 155-161.

[16] Erşahin, L. (2006): Quality and agronomic properties of sweet basil (Ocimum basilicum L.) grown in Diyarbakır ecological conditions. - C. U. Institute of Natural and Applied Sciences, Master thesis, Adana.

[17] Gairola, S., Shariff, N. M., Bhatt, A. (2010): Influence of climate change on production of secondary chemicals in high altitude medicinal plants: issues needs immediate attention. Journal of Medicinal Plants Research 4(18): 1825-1829.

[18] Gill, B., Randhawa, G. (1992): Effect of transplanting dates and stage of harvesting on the herb and oil yields of French basil (Ocimum basilicum L.). - Indian Perfumer, pp. 102-110.

[19] Hiltunen, R. (1999): Chemical composition of Ocimum species. - In: Basil-The Genus Ocimum. Harwood Academic Publishers, pp. 67-75.

[20] Jaćimović, G., Crnobarac, J., Marinković, B., Ninić-Todorović, J., Štetić, J. (2010): The yield and morphological properties of calendula and basil in relation to nitrogen fertilization. - Letopis naučnih radova Poljoprivrednog fakulteta 34(1): 69-79.

[21] Javanmardi, J., Ghorbani, E. (2012): Effects of chicken manure and vermicompost teas on herb yield, secondary metabolites and antioxidant activity of lemon basil (Ocimum $\times$ citriodorum Vis.). - Advances in Horticultural Science 26(3-4): 151-157.

[22] Kothari, S., Bhattacharya, A., Ramesh, S. (2004): Essential oil yield and quality of methyl eugenol rich Ocimum tenuiflorum Lf (syn. O. sanctum L.) grown in south India as influenced by method of harvest. - Journal of Chromatography A 1054(1-2): 67-72.

[23] Liang, X., Zhang, L., Natarajan, S. K., Becker, D. F. (2013): Proline mechanisms of stress survival. - Antioxidants \& Redox Signaling 19(9): 998-1011.

[24] Maggio, A., Roscigno, G., Bruno, M., De Falco, E., Senatore, F. (2016): Essential-oil variability in a collection of Ocimum basilicum L.(Basil) cultivars. - Chemistry \& Biodiversity 13(10): 1357-1368.

[25] Majkowska-Gadomska, J., Mikulewicz, E., Dobrowolski, A., Dziedzic, A. (2015): Ecotype and foliar fertilization with Florovit affect herbage yield and quality of greenhouse-grown basil (Ocimum basilicum L.). - Journal of Agricultural Science 7(4): 195.

[26] Markowitz, K., Moynihan, M., Liu, M., Kim, S. (1992): Biologic properties of eugenol and zinc oxide-eugenol: a clinically oriented review. - Oral Surgery, Oral Medicine, Oral Pathology 73(6): 729-737.

[27] Mirzajani, Z., Hadavi, E., Kashi, A. (2015): Changes in the essential oil content and selected traits of sweet basil (Ocimum basilicum L.) as induced by foliar sprays of citric acid and salicylic acid. - Industrial Crops and Products 76: 269-274.

[28] Moayedi, F., Kordi, S., Mehrabi, A., Dastborhan, S. (2020): Effect of different levels of nitrogen on yield, nitrate content, essential oil yield and some physiological traits in four cultivars of sweet basil (Ocimum basilicum L.). - Journal of Horticultural Science 34(2): 285-301.

[29] Mondello, L., Zappia, G., Cotroneo, A., Bonaccorsi, I., Chowdhury, J. U., Yusuf, M., Dugo, G. (2002): Studies on the essential oil-bearing plants of Bangladesh. Part VIII. Composition of some Ocimum oils $O$. basilicum L. var. purpurascens; $O$. sanctum L. green; $O$. sanctum L. purple; O. americanum L., citral type; $O$. americanum L., camphor type. - Flavour and Fragrance Journal 17(5): 335-340. 
[30] Morales, M. R., Simon, J. E. (1996): New basil selections with compact inflorescences for the ornamental market. - Progress in New Crops, pp. 543-546.

[31] Nacar, S., Tansi, S. (2000): Chemical components of different basil (Ocimum basilicum L.) cultivars grown in Mediterranean regions in Turkey. - Israel Journal of Plant Sciences 48(2): 109-112.

[32] NASA. (2021): Turkey Experiences Intense Drought. - Retrieved 03.02.2021 from https://earthobservatory.nasa.gov/images/147811/turkey-experiences-intense-drought.

[33] Nurzynska-Wierdak, R. (2007): Comparing the growth and flowering of selected basil [Ocimum basilicum L.] varieties. - Acta Agrobotanica 60(2): 127-131.

[34] Nurzynska-Wierdak, R. (2011): Sweet basil (Ocimum basilicum L.) flowering affected by foliar nitrogen application. - Acta Agrobotanica 64(1): 57-64.

[35] Nurzynska-Wierdak, R. (2013): Morphological and chemical variability of Ocimum basilicum L.(Lamiaceae). - Modern Phytomorphology 3: 115-118.

[36] Padalia, R. C., Verma, R. S., Chauhan, A., Goswami, P., Singh, V. R., Verma, S. K., Darokar, M. P., Singh, N., Saikia, D., Chanotiya, C. S. (2017): Essential oil composition and antimicrobial activity of methyl cinnamate-linalool chemovariant of Ocimum basilicum L. from Indi. - Records of Natural Products 11(2): 193.

[37] Patel, R. P., Singh, R., Saikia, S. K., Rao, B. R., Sastry, K., Zaim, M., Lal, R. K. (2015): Phenotypic characterization and stability analysis for biomass and essential oil yields of fifteen genotypes of five Ocimum species. - Industrial Crops and Products 77: 21-29.

[38] Paton, A., Harley, M., Harley, M. (1999): Ocimum: an overview of classification and relationships. Basil: the genus Ocimum. - Harwood, Amsterdam, pp. 1-38.

[39] Phippen, W., Simon, J. E. (2000): Anthocyanin inheritance and instability in purple basil (Ocimum basilicum L.). - Journal of Heredity 91(4): 289-296.

[40] Rao, B. R. R., Kothari, S. K., Rajput, D. K., Patel, R. P., Darokar, M. P. (2011): Chemical and biological diversity in fourteen selections of four Ocimum species. - Natural Product Communications 6(11): 1705-1710.

[41] Schulz, H., Schrader, B., Quilitzsch, R., Pfeffer, S., Krüger, H. (2003): Rapid classification of basil chemotypes by various vibrational spectroscopy methods. - Journal of Agricultural and Food Chemistry 51(9): 2475-2481.

[42] Sharma, M., Thakur, R., Sharma, M., Sharma, A. K., Sharma, A. K. (2020): Changing scenario of medicinal plants diversity in relation to climate change: a review. - Plant Archives 20(2): 4389-4400.

[43] Shen, T., Yu, H., Wang, Y.-Z. (2021): Assessing the impacts of climate change and habitat suitability on the distribution and quality of medicinal plant using multiple information integration: Take Gentiana rigescens as an example. - Ecological Indicators 123: 107376.

[44] Simon, J. E., Quinn, J., Murray, R. G. (1990): Basil: a source of essential oils. - In: Janick, J., Simon, J. E. (eds.) Advances in new crops. Timber Press, Portland, OR, pp. 484-489.

[45] Simon, J. E., Reiss-Bubenheim, D., Joly, R. J., Charles, D. J. (1992): Water stress-induced alterations in essential oil content and composition of sweet basil. - Journal of Essential Oil Research 4(1): 71-75.

[46] Sims, C. A., Juliani, H. R., Mentreddy, S., Simon, J. E. (2014): Essential oils in holy basil (Ocimum tenuiflorum L.) as influenced by planting dates and harvest times in North Alabama. - Journal of Medicinally Active Plants 2(3): 33-41.

[47] Sullivan, C. (2009): Food for Thought: The Science, Culture \& Politics of Food in Spring. - College Seminar 235(2).

[48] Suprasanna, P., Nikalje, G., Rai, A. (2016): Osmolyte accumulation and implications in plant abiotic stress tolerance. - In: Iqbal, N., Nazar, R. A., Khan, N. (eds.) Osmolytes and Plants Acclimation to Changing Environment: Emerging Omics Technologies. Springer, New Delhi. https://doi.org/10.1007/978-81-322-2616-1_1.

[49] Svecova, E., Neugebauerova, J. (2010): A study of 34 cultivars of basil (Ocimum L.) and their morphological, economic and biochemical characteristics, using standardized descriptors. - Acta Univ. Sapientiae, Alimentaria 3: 118-135. 
[50] Tahhami Zarandi, S., Rezvani Moghaddam, P., Jahan, M. (2014): Evaluation the effects of organic, biological and chemical fertilizers on morphological traits, yield and yield components of basil (Ocimum basilicum L.). - Iranian Journal of Field Crops Research 12(4): 543-553.

[51] Tansi, S., Nacar, S. (2000): First cultivation trials of lemon basil (Ocimum basilicum var. citriodorum) in Turkey. - Pakistan Journal of Biological Sciences 3(3): 395-397.

[52] Toncer, O., Karaman, S., Diraz, E., Tansi, S. (2017): Essential oil composition of Ocimum basilicum L. at different phenological stages in semi-arid environmental conditions. Fresenius Environ. Bull 26: 5441-5446.

[53] Tsasi, G., Mailis, T., Daskalaki, A., Sakadani, E., Razis, P., Samaras, Y., Skaltsa, H. (2017): The effect of harvesting on the composition of essential oils from five varieties of Ocimum basilicum L. cultivated in the island of Kefalonia, Greece. - Plants 6(3): 41.

[54] Varga, F., Carović-Stanko, K., Ristić, M., Grdiša, M., Liber, Z., Šatović, Z. (2017): Morphological and biochemical intraspecific characterization of Ocimum basilicum L. Industrial Crops and Products 109: 611-618.

[55] Vieira, R. F., Simon, J. E. (2000): Chemical characterization of basil (Ocimum spp.) found in the markets and used in traditional medicine in Brazil. - Economic Botany 54(2): 207216.

[56] Vieira, R. F., Simon, J. E. (2006): Chemical characterization of basil (Ocimum spp.) based on volatile oils. - Flavour and fragrance journal 21(2): 214-221.

[57] Vieira, P. R., de Morais, S. M., Bezerra, F. H., Ferreira, P. A. T., Oliveira, Í. R., Silva, M. G. V. (2014): Chemical composition and antifungal activity of essential oils from Ocimum species. - Industrial Crops and Products 55: 267-271.

[58] Viña, A., Murillo, E. (2003): Essential oil composition from twelve varieties of basil (Ocimum spp.) grown in Colombia. - Journal of the Brazilian chemical society 14(5): 744749.

[59] Walters, K. J., Currey, C. J. (2019): Growth and development of basil species in response to temperature. - HortScience 54(11): 1915-1920.

[60] Yancey, P. H. (2005): Organic osmolytes as compatible, metabolic and counteracting cytoprotectants in high osmolarity and other stresses. - Journal of Experimental Biology 208(15): 2819-2830. 